\title{
Monetary reward increases attentional effort in the flanker task
}

\author{
RONALd HÜBNER ANd JAN SCHLÖSSER \\ Universität Konstanz, Konstanz, Germany
}

\begin{abstract}
An important question is whether monetary reward can increase attentional effort in order to improve performance. Up to now, evidence for a positive answer is weak. Therefore, in the present study, the flanker task was used to examine this question further. Participants had to respond sooner than a certain deadline in a flanker task. One group of participants received a performance-contingent monetary reward, whereas the other group earned a fixed amount of money. As a result, monetary reward significantly improved performance in comparison with the control group. The analysis of speed-accuracy trade-off functions revealed that monetary reward increased attentional effort, leading to an enhanced quality of stimulus coding. Little evidence was found that reward also improved selective spatial attention.
\end{abstract}

Because cognitive capacity is limited, it is economical for an organism to mainly allocate its resources to mental activity that is important for its survival or relevant for attaining its current goals. It is widely assumed that two attentional control systems serve this objective. The first system encompasses bottom-up mechanisms that automatically allocate attentional resources to the processing of perceptually (see, e.g., Wolfe, Butcher, Lee, \& Hyle, 2003) or emotionally (e.g., Schupp et al., 2004) salient stimuli. The second system consists of top-down mechanisms that can be used for deliberately allocating mental resources to tasks and to the processing of relevant stimuli that lack saliency. A crucial difference between these systems is that bottom-up control proceeds automatically, whereas top-down control requires effort. An important question in this respect is whether effort for top-down control can be increased, and if so, by what mechanism. Formerly, it was thought that attentional effort could be mobilized only by task demands (Kahneman, 1973). In contrast, more recent studies have suggested that effort can also be increased by motivational factors (cf. Sarter, Gehring, \& Kozak, 2006). However, as Sarter et al. remarked, the experimental evidence for motivational effects is still weak and "in stark contrast to the very common experience of being motivated to increase 'attentional effort' in order to perform better" (p. 147). Thus, the aim of the present study was to further investigate how motivation mobilizes attentional effort.

The weak evidence for motivational effects on attentional effort can be seen paradigmatically in studies that have tried to increase performance via monetary reward (for overviews, see Bonner \& Sprinkle, 2002; Camerer \& Hogarth, 1999; Jenkins, Mitra, Gupta, \& Shaw, 1998). The resulting effects were often small or absent (e.g., Small et al., 2005; Taylor et al., 2006), and in some studies even negative (e.g., Mobbs et al., 2009). If one considers the few positive studies, it still often remains open whether the performance was indeed increased by attentional effort, and if so, exactly which mechanisms were involved. The difficulty is that an increase in performance can result from various mechanisms. For instance, monetary reward can speed up stimulus coding and/or motoric responding. The saved time can then be spent to extend the response selection phase, which, in turn, improves sensitivity (see below). Thus, even though results have shown that monetary reward increases perceptual sensitivity (e.g., Engelmann, Damaraju, Padmala, \& Pessoa, 2009; Engelmann \& Pessoa, 2007; Kiss, Driver, \& Eimer, 2009), it cannot automatically be concluded that this increase was due to improved attention.

That monetary reward can affect specific attentional mechanisms has been shown in a study by Della Libera and Chelazzi (2006). They found that monetary reward can affect negative priming, a mechanism that modulates the subsequent deployment of attention (see also Della Libera \& Chelazzi, 2009). However, in their study, reward was completely decoupled from actual behavioral performance and was delivered arbitrarily. Moreover, even though negative priming was increased after a high reward, responses were faster after low rewards.

Thus, altogether, the results in this area are inconclusive. In studies showing that reward improves performance, the involved mechanisms remain unclear, and in studies demonstrating that reward can affect specific mechanisms, it remains open whether these modulations can really improve performance. Therefore, in the present study, we wanted to further investigate whether monetary reward can increase performance, and if so, which mechanisms are involved. Possible mechanisms should be distinguished by examining speed-accuracy trade-off 
functions (SATFs), which describe the relation between the mean response time (RT) for a certain deadline condition and the resulting accuracy. A specific question was whether monetary reward can improve selective visual attention.

For this objective, we used the flanker task (Eriksen \& Eriksen, 1974), which is one of the main paradigms for investigating selective visual attention. In this task, participants have to decide whether a target stimulus belongs to one category or to another. Moreover, the target is flanked by irrelevant stimuli. In our case, the flankers could be either incongruent or neutral with respect to the required response to the target. Usually, responses to targets accompanied by incongruent flankers are slower and less accurate than those to targets accompanied by neutral flankers. The size of this difference in performance, which will be called the flanker effect (FE), indicates the efficiency of selective attention. A small effect is interpreted to indicate that attention is narrowly focused on the target stimulus, whereas a large effect indicates a wide focus that also encompasses - at least to some extent- the flankers.

We tried to modulate attentional effort by applying two reward conditions. Participants in one condition received monetary reward, whereas those in the other conditions merely received symbolic reward. Because the basic performance measures in the flanker task - that is, RT and accuracy - can mutually be traded, both measures had to be taken into account for rewarding. Therefore, our participants had to respond before a certain deadline and received a positive payoff of 10 points for each correct response and a penalty of -10 points for each commission error (wrong response within deadline). For each deadline error (missed deadline), there was a penalty of -20 points. At the end of each trial, the updated sum of the accumulated points was shown as part of a general feedback. The participants in the monetary reward condition were informed that at the end of the experiment the final sum of points would be converted into money, whereas the participants in the symbolic reward condition received a flat rate for their participation; that is, their performance-dependent reward was purely symbolic.

SATFs were obtained by changing the deadline in two steps in the course of the experiment. In order to be able to differentiate between the various mechanisms that could be affected by monetary reward, we computed corresponding theoretical SATFs (see Figure 1) by means of a diffusion model (cf. Ratcliff \& Rouder, 1998), which belongs to the widely applied class of sequential sampling models. The diffusion model assumes that response selection proceeds by accumulating evidence at a certain rate for the two response alternatives. If the evidence reaches a decision boundary for either the one alternative or the other, the corresponding response is executed. In this model, speed can be traded for accuracy by simply changing the boundaries, which is assumed to be under voluntary control. If, for instance, response speed must be increased to meet a shorter deadline, then the boundaries can be lowered, so that less evidence is necessary for a decision. This leads to faster responses, but also decreases accuracy, because the decisions are now based on less evidence. Thus, for a given rate of evidence accumulation, the corresponding SATF can be obtained by varying the decision boundaries.

These theoretical calculations allowed us to predict how the SATF would change for the various effects that an increase of motivation due to monetary reward could

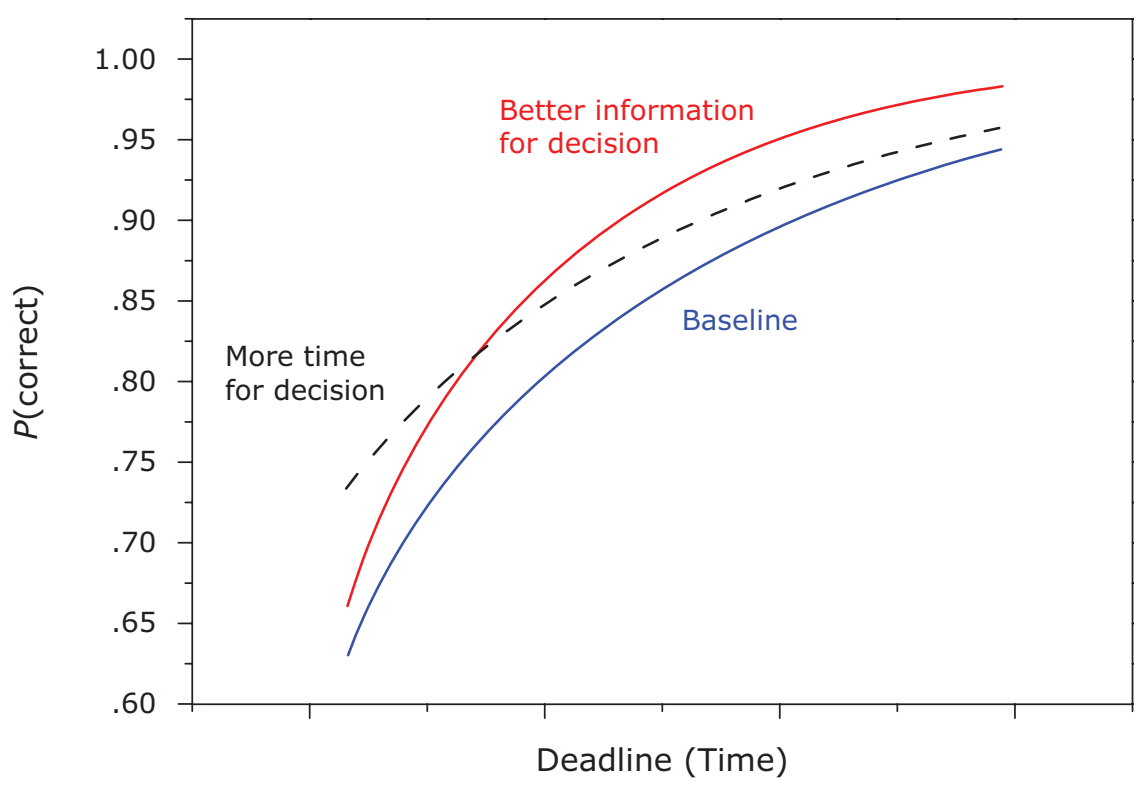

Figure 1. Theoretical speed-accuracy trade-off functions (SATFs). The two upper SATFs show possible effects of increased mental effort on the performance, relative to a baseline condition (see text for details). 
potentially have. By comparing the empirical with the theoretical SATFs, we were then able to draw conclusions regarding the underlying mechanisms that are affected by the increase in effort.

As mentioned, one effect could be that an increased effort mobilized by monetary reward speeds up stimulus coding and/or motoric responding. This saves time that can then be used to extend the response selection phase. As a result, accuracy is increased for a given deadline, relative to a baseline condition. As can be seen in Figure 1 (see the "More time for decision" curve), a characteristic of this effect is that the increase is relatively large for short deadlines, but then decreases with an increasing deadline. Thus, if monetary reward, compared with symbolic reward, simply speeds up nondecisional processing, then the difference in accuracy between the corresponding SATFs should decrease with an increasing deadline.

Another and more specific effect of monetary reward could be that it mobilizes attentional resources that improve the quality of sensory coding (cf. Goard \& Dan, 2009; Sarter et al., 2006), which leads to an increased rate of evidence accumulation. As can be seen in Figure 1 (see the "Better information for decision" curve in Figure 1), in this case the improvement in accuracy would increase with the deadline for the lower range of accuracies and then almost remain constant until it slightly decreases at the higher end.

Finally, an even more specific effect of monetary reward would be to improve spatial selectivity - for example, by focusing more narrowly on the target - thereby reducing the effect of the flankers. In order not to discourage our participants from using such a strategy, we excluded congruent flankers in the present experiment. Because congruent flankers activate the correct response, focusing of spatial attention narrowly on the target would not be required for these stimuli and, therefore, might not be an effective strategy. If only incongruent and neutral flankers can occur, then a narrow focus should always improve performance, or at least not impair it. Thus, if monetary reward improves spatial selectivity, then performance should be improved mainly for stimuli with incongruent flankers, whereas that for stimuli with neutral flankers should remain relatively unaltered. Consequently, the difference in accuracy between the SATFs for incongruent and neutral flankers should be reduced. This should be reflected by a smaller FE for the monetary reward group.

\section{METHOD}

\section{Participants}

One hundred four students from the Universität Konstanz were randomly assigned to the monetary reward condition (mean age 22.5 years; 16 males), or to the symbolic-reward condition (mean age of 23.2 years; 12 males). Members of the symbolic reward group were paid $€ 8$ per hour, whereas members of the monetary reward group were paid a base payment of $€ 6$ per hour and were informed in advance that they could additionally earn up to $€ 5$, depending on the points gained in the experiment. All participants started with a "capital" of 1,000 points.

\section{Apparatus and Stimuli}

Stimuli were presented on an 18 -in. color monitor with a resolution of $1,280 \times 1,024$ pixels and a refresh rate of $60 \mathrm{~Hz}$. Participants responded by pressing one of two buttons on a computer mouse. Stimulus presentation as well as response registration was controlled by the same personal computer.

Target items were numerals from 2 to 9 . Incongruent stimuli were constructed by using response-incompatible numerals as flankers. For neutral stimuli the characters $\$, \&$, ?, and \# served as flankers. Target and flankers were arranged horizontally, and the target was presented at the center of the screen. Each single character extended a visual angle of $0.9^{\circ}$ horizontally and $1.27^{\circ}$ vertically; the spacing between the items (center to center) was $1.27^{\circ}$ of visual angle. Stimuli were presented in white on a black background.

\section{Procedure}

Participants were seated at a viewing distance of approximately $45 \mathrm{~cm}$ in front of the screen. A trial started with the presentation of a fixation cross at the center of the screen for $400 \mathrm{msec}$. After a cue stimulus interval of $600 \mathrm{msec}$, the stimulus array appeared for $165 \mathrm{msec}$. The screen remained blank until the participant responded. After the response, a feedback screen appeared for $1,300 \mathrm{msec}$. After a blank screen for $1,000 \mathrm{msec}$, the next trial started (see Figure 2). The task of the participants was to indicate the parity of the target numeral by pressing a corresponding response button with the index or middle finger of the right hand. Response errors were signaled by a short sound. Half of the participants started by completing three blocks in which the response deadline was $650 \mathrm{msec}$. Subsequently, there were three blocks with a deadline of $525 \mathrm{msec}$, followed by three blocks with a deadline of $450 \mathrm{msec}$. To counterbalance possible practice effects, the other half of the participants worked through the reversed order of deadlines. All blocks of 64 trials each were administered in a 1-h session.

Feedback. After each response, a feedback screen was displayed for 1,300 msec (see Figure 2) informing the participants about their performance on the current trial. In addition to the deadline in the current block, the RT was shown. It was displayed in green if the response was correct and occurred before the deadline, in red if the response was an error but occurred before the deadline, and in yellow if the response missed the deadline, regardless of whether it was correct or not. The participants were also informed about the current sum of their points (see Figure 2). After each block, an additional feedback screen was shown for maximally $60 \mathrm{sec}$, displaying information about their overall performance. It informed about the points gained so far (accumulated over all blocks), the mean RT in the last block, the error rate in the last block, and the percentage of missed deadlines in the last block.

Participants received 10 points when their response was earlier than the deadline and correct. If their response was earlier than the deadline but incorrect, they lost 10 points. If they missed the deadline, they lost 20 points. The participants in the reward group knew from the beginning that the possible maximal amount of money they could earn was $€ 5$, but they were not told before the end of the experiment that 10 points were worth 1 Euro-cent.

\section{RESULTS}

The mean latencies of correct responses (deadline errors were not excluded) were entered into a three-factor ANOVA on the between-subjects factor reward (monetary or symbolic) and the within-subjects factors deadline (long, medium, or short), and flanker type (neutral or incongruent). Accuracy was analyzed by subjecting percentage correct values to ANOVAs of the same type as that for the latencies (it should be mentioned that analyz- 


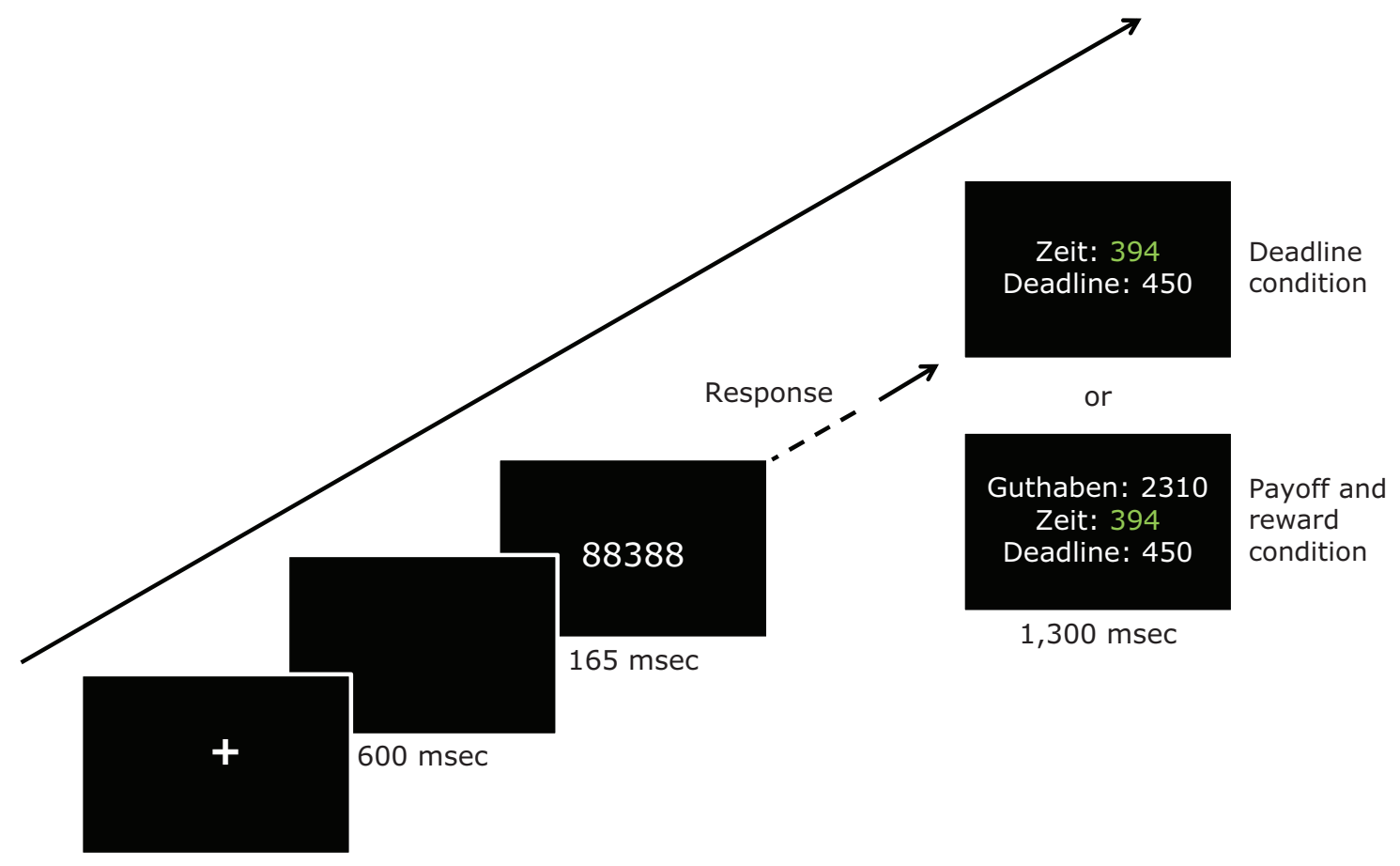

$400 \mathrm{msec}$

Figure 2. Sequence of events on a trial. After each response a feedback screen was shown. All participants were informed about their response time ("Zeit") in the current trial and the deadline in the current block. Accuracy was signaled by the color of the response time feedback: A green number meant ok, a red number meant commission error, and a yellow number meant deadline error. Participants in the payoff and reward groups additionally received information about their accumulated points ("Guthaben").

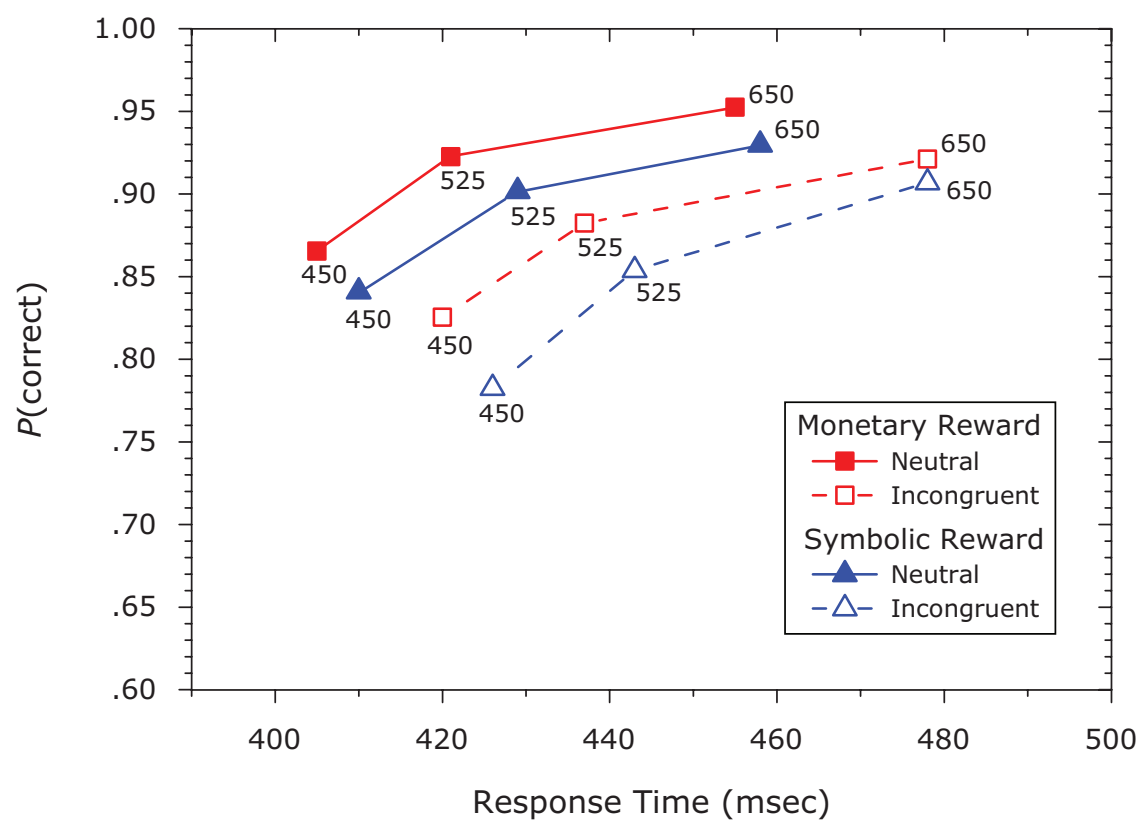

Figure 3. Speed-accuracy trade-off functions of the two groups. The numbers at the data points indicate the corresponding deadline. 
ing $d^{\prime}$ measures revealed a similar pattern of results). The corresponding SATFs are shown in Figure 3.

\section{Response Times}

The analysis revealed highly significant main effects of deadline $[F(2,204)=106, p<.001]$ and flanker type $[F(1,102)=328, p<.001]$. The reward factor was not significant, but the trend of $5 \mathrm{msec}$ was toward faster responses for the monetary reward condition. There was also a significant two-way deadline $\times$ flanker type interaction $[F(2,204)=9.93, p<.001]$. The FE increased with an increasing deadline $(14,17$, and $23 \mathrm{msec})$.

\section{Accuracy}

The analysis revealed significant main effects of deadline $[F(2,204)=144, p<.001]$, flanker type $[F(1,102)=$ $159, p<.001]$, and reward $[F(1,102)=6.20, p<.05]$. Most importantly, accuracy was higher for monetary reward than for symbolic reward ( $89.5 \%$ vs. $86.9 \%)$. Concerning possible differences in FE between the reward conditions, although the FE was numerically smaller for the monetary reward condition than for the symbolic reward condition $(3.26 \%$ vs. $3.85 \%)$, the corresponding two-way reward $\times$ flanker type interaction was far from significant $[F(1,102)=0.82, p=.37]$.

However, there was a significant two-way deadline $\times$ flanker type interaction $[F(2,204)=6.79, p<.01]$. The FE decreased with an increasing deadline $(4.92 \%, 4.40 \%$, $2.71 \%)$. Moreover, there was the trend $[F(2,204)=2.34$, $p=.10]$ that this decrease differed between the reward conditions (cf. Figure 3). A separate analysis of the two reward conditions revealed that the decrease in FE $(5.84 \%$, $4.79 \%, 2.28 \%)$ was highly significant $[F(2,102)=8.51$, $p<.001]$ for the symbolic reward condition, but practically negligible $(4.01 \%, 4.02 \%, 3.14 \%)$ for the monetary reward condition $[F(2,102)=0.64, p=.53]$.

\section{DISCUSSION}

Our results show that accuracy decreased monotonically with a decreasing deadline. This demonstrates that typical SATFs (see Figure 3) were obtained in our flanker task. Moreover, we also found that participants who received a performance-contingent monetary reward had a significantly higher accuracy than did participants who earned a fixed amount of money and whose performance was merely rewarded symbolically. Thus, our results are in line with the few studies showing that monetary reward can improve performance (e.g., Engelmann et al., 2009; Engelmann \& Pessoa, 2007; Kiss et al., 2009). However, given our theoretical considerations, we can not only be confident that monetary reward indeed increased attentional effort, but also conclude which mechanisms were involved and which were not.

If we consider the SATFs for responses to the neutral stimuli, then we see that monetary reward improved accuracy by a similar amount for all deadlines. According to our reasoning based on the theoretical SATFs in the in- troduction, this implies that reward mobilized attentional resources that enhanced the quality of sensory coding and of corresponding cortical representations (cf. Goard \& Dan, 2009; Sarter et al., 2006). This higher quality led to a higher rate of evidence accumulation, which improved the overall accuracy.

The fact that the FE did not differ substantially between the reward conditions indicates that the improvement of the sensory coding was unselective. That is, monetary reward did not reduce the spatial attentional focus. In this case, the FE should have been significantly smaller in that condition. The results merely indicate that the FE remained constant across deadlines in the monetary reward condition, whereas it increased with a decreasing deadline in the symbolic reward condition. The latter result could be interpreted in the sense that in the condition with a short deadline, the participants not only lowered their decision criterion, but also relaxed their spatial selectivity. With monetary reward, though, selectivity was maintained also for short deadlines. However, this interpretation is rather speculative.

Thus, altogether, our results provide clear evidence for the hypothesis that performance-contingent monetary reward can increase attentional effort to perform better. Obviously, reward mobilizes attentional resources that improve stimulus coding, which, in turn, increases accuracy. Concerning selectivity, we cannot definitively rule out that monetary reward also increased selective spatial attention. However, if at all, the increase occurred under rather specific conditions (short deadline), and its contribution to the overall improvement of accuracy was relatively small.

\section{AUTHOR NOTE}

Correspondence should be addressed to R. Hübner, Universität Konstanz, Fachbereich Psychologie, Fach D29, D-78457 Konstanz, Germany (e-mail: ronald.huebner@uni-konstanz.de).

Note-Accepted by Cathleen M. Moore's editorial team.

\section{REFERENCES}

Bonner, S., \& Sprinkle, G. B. (2002). The effects of monetary incentives on effort and task performance: Theories, evidence, and a framework for research. Accounting, Organizations \& Society, 27, 303-345.

Camerer, C. F., \& Hogarth, R. M. (1999). The effects of financial incentives in experiments: A review and capital-labor-production framework. Journal of Risk \& Uncertainty, 19, 7-42.

Della Libera, C. D., \& Chelazzi, L. (2006). Visual selective attention and the effects of monetary rewards. Psychological Science, 17, 222-227.

Della Libera, C. D., \& Chelazzi, L. (2009). Learning to attend and to ignore is a matter of gains and losses. Psychological Science, 20, 778-784.

Engelmann, J. B., Damaraju, E., Padmala, S., \& Pessoa, L. (2009). Combined effects of attention and motivation on visual task performance: Transient and sustained motivational effects. Frontiers in Human Neuroscience, 3, 1-16.

Engelmann, J. B., \& Pessoa, L. (2007). Motivation sharpens exogenous spatial attention. Emotion, 7, 668-674.

ERIKSEN, B. A., \& ERIKSEN, C. W. (1974). Effects of noise letters upon the identification of a target letter in a nonsearch task. Perception \& Psychophysics, 16, 143-149. 
GOARD, M., \& DAN, Y. (2009). Basal forebrain activation enhances cortical coding of natural scenes. Nature Neuroscience, 12, 1440-1447.

Jenkins, G. D., Mitra, A., Gupta, N., \& Shaw, J. D. (1998). Are financial incentives related to performance? A meta-analystic review of empirical research. Journal of Applied Psychology, 83, 777-787.

Kahneman, D. (1973). Attention and effort. Englewood Cliffs, NJ: Prentice Hall.

Kiss, M., Driver, J., \& Eimer, M. (2009). Reward priority of visual target singletons modulates event-related potential signatures of attentional selection. Psychological Science, 20, 245-251.

Mobbs, D., Hassabis, D., Seymour, B., Marchant, J. L., Weiskopf, N., Dolan, R. J., ET AL. (2009). Choking on the money: Reward-based performance decrements are associated with midbrain activity. Psychological Science, 20, 955-962.

RATCLIFF, R., \& Rouder, J. N. (1998). Modeling response times for two-choice decisions. Psychological Science, 9, 347-356.

Sarter, M., Gehring, W. J., \& KozaK, R. (2006). More attention must be paid: The neurobiology of attentional effort. Brain Research Reviews, 51, 145-160.
Schupp, H. T., Cuthbert, B. N., Bradley, M. M., Hillman, C. H., HAMm, A. O., \& LANG, P. J. (2004). Brain processes in emotional perception: Motivated attention. Cognition \& Emotion, 18, 593-611.

Small, D. M., Gitelman, D., Simmons, K., Bloise, S. M., Parrish, T., \& Mesulam, M. M. (2005). Monetary incentives enhance processing in brain regions mediating top-down control of attention. Cerebral Cortex, 15, 1855-1865.

Taylor, S. F., Martis, B., Fitzgerald, K. D., Welsh, R. C., Abelson, J. L., Liberzon, I., ET AL. (2006). Medial frontal cortex activity and loss-related responses to errors. Journal of Neuroscience, 26, 40634070.

Wolfe, J. M., Butcher, S. J., Lee, C., \& Hyle, M. (2003). Changing your mind: On the contributions of top-down and bottom-up guidance in visual search for feature singletons. Journal of Experimental Psychology: Human Perception \& Performance, 29, 483-502.

(Manuscript received May 17, 2010; revision accepted for publication July 8,2010 .) 